\title{
DETECTING LOWER LIMB RANGE OF MOTION USING LOW COST ACCELEROMETERS AND GYROSCOPES
}

\author{
Michal Čekan, Miloš Musil
}

Slovak University of Technology in Bratislava, Faculty of Mechanical Engineering, Nam. Slobody 17,812 31, michal.cekan@stuba.sk

Keywords: Range of Motion, Gait analysis, Accelerometers, Gyroscopes, inertial measuring units

\begin{abstract}
Understanding gait parameters of patients with limited mobility help specialists monitor, predict, and even control the progression of patient specific pathologies. Accurately determining gait parameters such as joint range of motion (ROM) often involves expensive equipment that typically requires a clinical setting and specially trained personnel. Added to this limitation is the psychological effects of patients that are required to perform in a clinical setting, which may not always reflect their actual day to day gait. The presented article implements accelerometers and gyroscopes, also known as inertial measuring units (IMU), mounted at key anatomical locations of the lower limbs to determine knee ROM. Measurements were performed in tandem with optical motion capture (OMC) which is considered the gold standard for gait analysis. The results show that IMU's have comparable accuracy to OMC measurements providing an economical alternative to expensive clinical gait analysis that is not restricted to a clinical setting.
\end{abstract}

\section{INTRODUCTION}

Gait is an important part of everyday human activity. Healthy gait provides a comfortable means for humans to be mobile. Patients that suffer from lower limb or gait related pathologies are affected both physically and psychologically. A little over the past decade, New methods for analyzing gait have become available, namely the OMC method. The OMC method offers accurate measurements that record important data that describe a patient's specific gait which can be used to diagnose problem areas when compared to healthy gait data. Although the OMC method is very accurate, it does have some limitations. OMC is still expensive and typically requires a clinical setting and special environmental conditions in order to record reliable data, and although some new systems claim to be "mobile" they require the movement of bulky equipment to the measurement location, which is not very practical for clinicians. This work introduces a low cost system with comparable accuracy to the OMC method using accelerometers and gyroscopes applied to the measurement of healthy lower limb gait. [1]

\section{METHOD}

Accelerometers offer highly sensitive measurements that can be used for tilt sensing [2]. However, due to their high sensitivity, measurements are typically saturated with noise and only work reliably in near static measurements. Gyroscopes on the other hand are less prone to noise and can be used in a more dynamic environment, but suffer from a phenomena known as drift over longer periods of measurement. Therefore, an accelerometer and gyroscope are mutually 
dependent on each other as one compensates for the shortcomings of the other. Fusing the measurements from both sensors and implementing the well-known Kalman filter to obtain the best estimate about the state of the system was tested for validity [3]. Its application can be described as follows:

-Predict the current state based on previous state and gyroscope measurements:

$$
x_{k=1}=F_{k} x_{k=1}+B_{k}, \theta_{k=1}+w_{k}
$$

Where $F$ is the state transition and B is the input model multiplied by the control (angular rate $\theta_{\mathrm{k}}$ )

as well as the expected Gaussian noise $w_{k}$ of the measurements.

-Measure and update:

$$
z_{\mathrm{x}}=H_{\mathrm{s}} x_{\mathrm{x}}+v_{\mathrm{x}}
$$

(2)

Where $H$ is the observation model and $v_{k}$ is the measurement noise which is also Gaussian.

The estimate then becomes the following linear function:

$$
x_{\text {ist }}=x_{\mathrm{R}}+R\left(\mathrm{R}_{\mathrm{R}}-x_{\mathrm{R}-1}\right)
$$

Where $\mathrm{K}$ is known as the Kalman gain used to correct the estimate based on the difference between actual and predicted measurements.

\section{MEASURMENTS}

The IMU was constructed by using a 3 axis $\pm 5 \mathrm{~g}$ analog accelerometer from analog devices. It was mounted orthogonally to a 2 axis gyroscope (LPR510AL from ST microelectronics) creating a 5 degree of freedom IMU. Measurements were given in $\mathrm{mV}$ recorded in .txt format by means of an analog to digital converter and Signal express software from National instruments. The hardware can be seen in fig. 1. The raw data was imported into Matlab software where it was converted to usable variables and implemented into a Kalman filter script. The validity of the measurements was provided by measuring in tandem with OMC methods which is currently considered the gold standard for gait analysis. The OMC laboratory was provided by the University of Olomouc, faculty of physical culture, Czech Republic. OMC measurements were provided from Vicon post processing in .txt format so that they could compare results directly in Matlab. A healthy female patient was chosen to perform 20 gait measurements each consisting of approximately four gait cycles. Specific OMC marker placement as well as IMU placement can be seen in fig. 2 . 


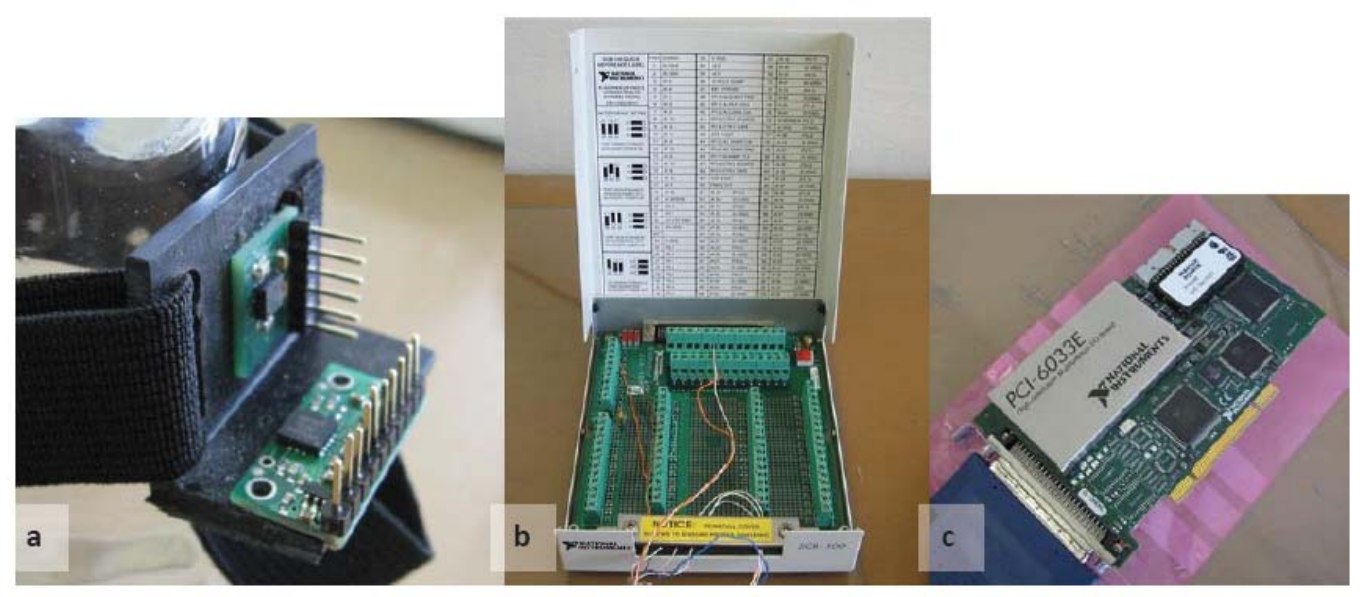

Figure 1: $a$ - accelerometer and gyroscope (IMU) mounted orthogonally on ABS chassis. $b-64$ channel breakout board, $c-A D C$

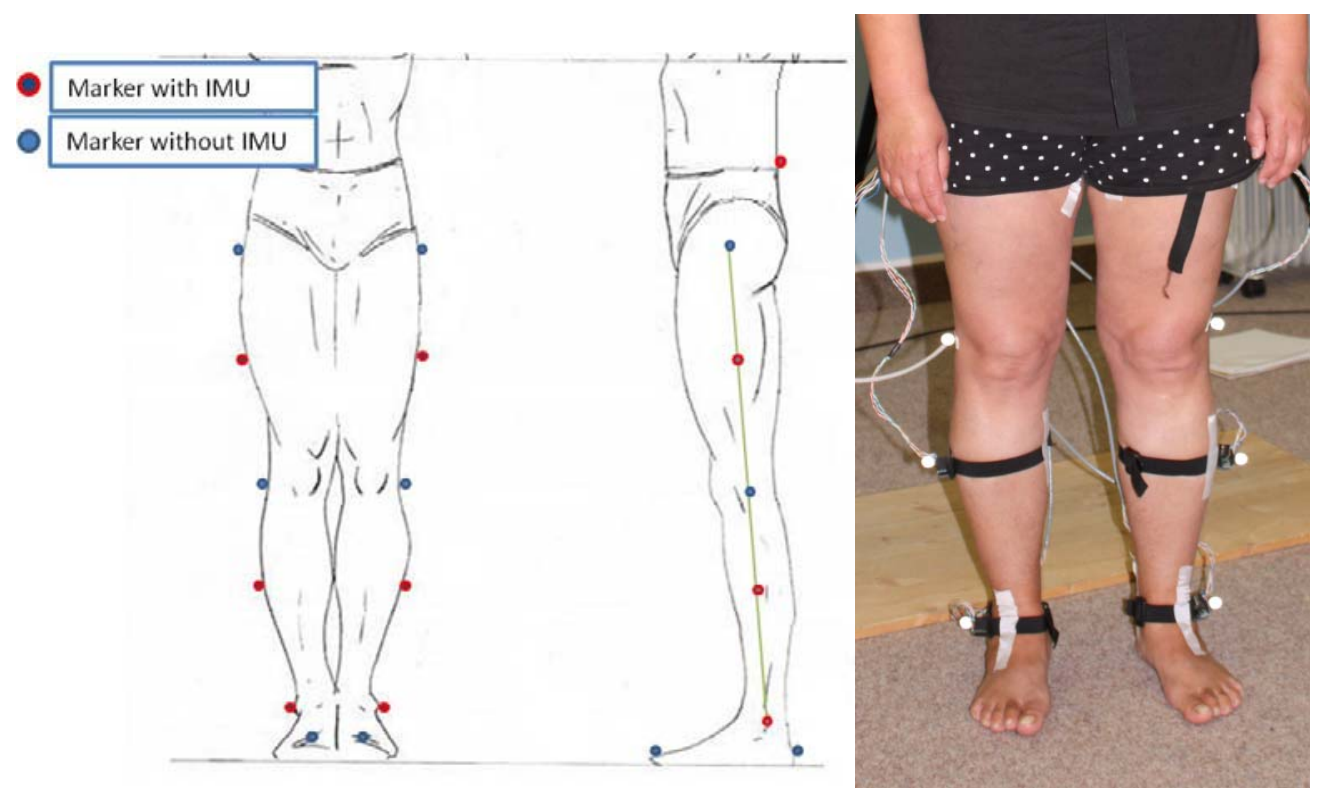

Figure 1: Sensor and marker placement on lower limbs of patient

\section{RESULTS}

The focus of this paper was to show the results obtained for knee ROM. It was obtained by subtracting the thigh angle from the shank angle. Of the 20 gait measurements, four gait cycles at a time where processed and the correlation between OMC and IMU measurements was calculated for sagittal plane knee ROM by: 


$$
R_{c}=\frac{n \sum x y-\left(\sum x\right)\left(\sum y\right)}{\sqrt{n\left(\sum x^{2}\right)-\left(\sum x\right)^{2}} \sqrt{n\left(\sum y^{2}\right)-\left(\sum y\right)^{2}}}
$$

where $n$ are the number of samples, $x$ is data from the IMU and $y$ is data from the OMC measurements. Values close to 1 indicate high levels of correlation between measurements. A visual comparison between results from IMU and OMC measurements can be seen in fig. 2

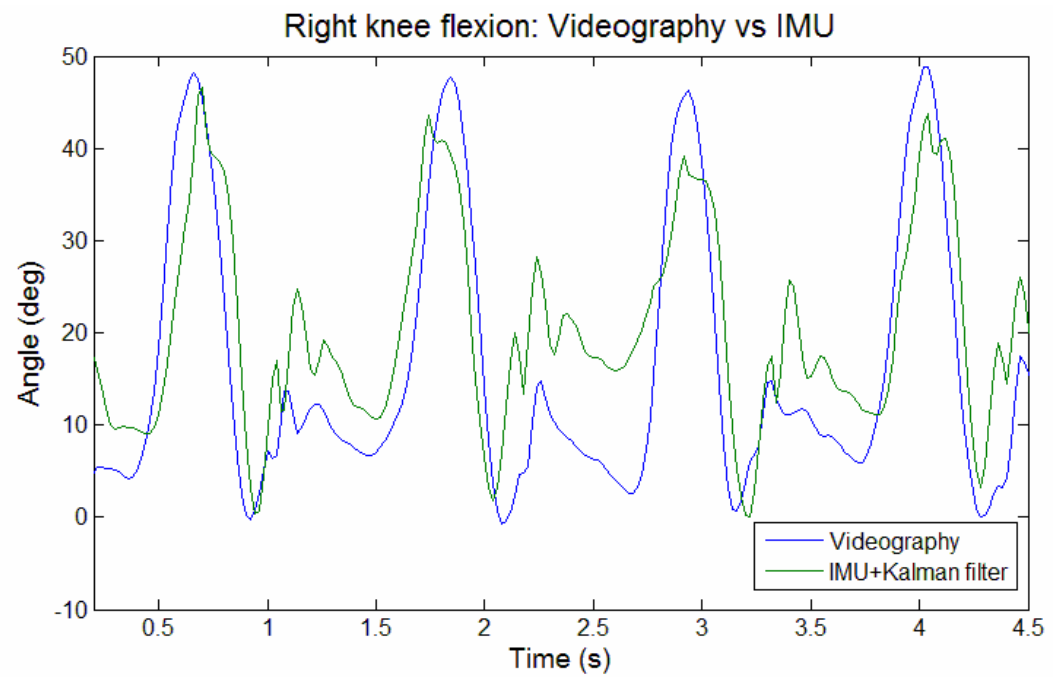

Figure 2: Comparison of right knee flexion angles recorded by OMC and IMU method

The lowest correlation through 20 measurements was found to be 0.883 while the highest correlation through the same set of data was 0.939 .

\section{CONCLUSION}

Although correlation between measurements is high, it is necessary to discuss the limiting factors of the current system. The developed IMU requires external connection through Category 5 ethernet cable to send signals to the ADC. Apart from being cumbersome, these cables interfere with the free movement of the IMU, future designs should incorporate an ADC and button battery directly on the IMU itself to eliminate any external connections. It would be beneficial to determine the joint angle in other planes as well to verify the accuracy for smaller ROM. Despite all of these shortcomings, and given the early developmental stage of this work, the use of IMU's as a cheap and portable alternative to $\mathrm{OMC}$ analysis is promising.

\section{Acknowledgment}

This project could not have been performed without the help of Zdenek Svoboda who graciously provided his optical motion capture laboratory at the University of Olomouc, faculty of physical culture. This paper was funded by the Vega program 1/0197/12. 


\section{REFERENCES}

[1] Čekan, Michal. An Alternative Approach for Gait Analysis Using Inertial Measuring Units. Dissertation thesis. Bratislava: SjF STU, 2012.

[2] Luinge, H.J., and Veltink, P.H. Measuring Orientation of Human Body Segments Using Miniature Gyroscopes and Accelerometers. In: Med. Biol. Eng. Comput., Vol. 43(2): 273-282, 2005.

[3] Marmion, M. Airborne attitude estimation using a Kalman filter UNIS. The University Centre of Svalbard, Longyearbyen, Norway, 2006.

[1] Hučko B., Čekan M., Šoltés L. Gait analysis using IMU sensors, In VII Congreso Bolivariano de Ingenieria Mecanica [elektronický zdroj] : Cusco, October 23-25, 2012. Lima: Universidad Católica del Perú, 2012, s. 7.

[3] Teunis, C., Scheffer, C. Benchmarking of a full-body inertial motion capture system for clinical gait analysis. Vancouver, British Columbia, Canada, 2008. 